\title{
The need for a large-scale, integrated approach to Ocean World Modeling
}

\section{Submitted by:}

William Farrell,NASA/Goddard Space Flight Center, 301-286-4446, William.M.Farrell@nasa.gov * Orenthal Tucker, NASA/Goddard Space Flight Center* Marc Neveu, NASA/Goddard Space Flight Center* Dina Bower, NASA/Goddard Space Flight Center * Lynnae Quick, NASA/Goddard Space Flight Center* Joseph Renaud, NASA/Goddard Space Flight Center* Robert Tyler, NASA/Goddard Space Flight Center* Louis-Alexandre Couston, British Antarctic Survey, Cambridge UK Conor Nixon, NASA/Goddard Space Flight Center* Paul Romani, NASA/Goddard Space Flight Center* Reggie Hudson, NASA/Goddard Space Flight Center* Cesare Grava, Southwest Research Institute Denis Felikson, NASA Postdoc Program, NASA/Goddard Space Flight Center* Erica Nathan, Brown University Geronimo Villanueva, NASA/Goddard Space Flight Center* Veronica Allen, NASA Postdoc Program, NASA/Goddard Space Flight Center* Shawn Domagal-Goldman, NASA/Goddard Space Flight Center* Jennifer Eigenbrode, NASA/Goddard Space Flight Center* Bethany Theiling, NASA/Goddard Space Flight Center* Gordon Bjoraker, NASA/Goddard Space Flight Center* Krista Soderlund, University of Texas, Austin

*Members of NASA/GSFC's Ocean worlds Science, Exploration and ANalog (OSEAN) team Italics indicates key contribution to paper as part of the principal writing team 


\section{Motivation}

The past decade has seen greatly enhanced scientific and political interest in new generation studies of the now known-to-benumerous Ocean Worlds in our solar system. These bodies include Jupiter's moons Europa, Ganymede, and Callisto, Saturn's moons Enceladus (Figure 1) and Titan, Neptune's moon Triton, and possibly the dwarf planets Pluto and Ceres. This enhanced interest has not only led to the build of the Europa Clipper (EC) Flagship mission (as

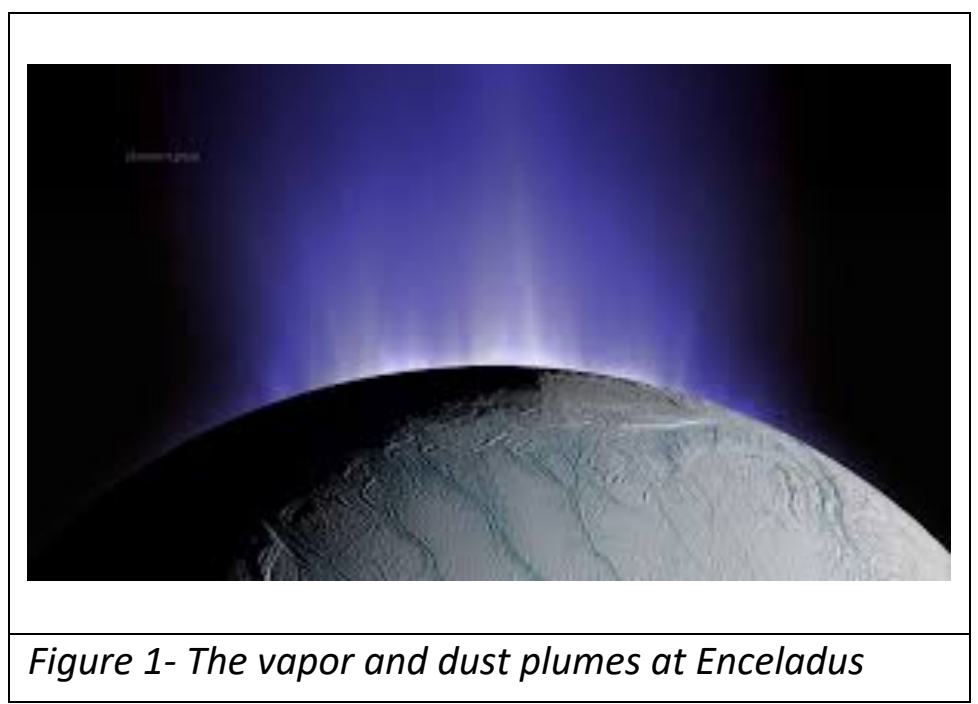
recommended by the last Decadal Survey, Vision \& Voyages), but also NASA considering the real and distinct possibility of an augmented Europa Lander capability to further examine the astrobiological potential of this moon. Since V\&V, Titan and Enceladus have been added to the New Frontiers target list. Thus, in response to NF-4, four concepts were proposed to examine the habitability and/or search for life on these ocean worlds. The selected NF-4, Dragonfly, is a rotorcraft going to Titan specifically designed to assess the astrobiological potency, hinted at by Cassini/Huygens, at multiple locations of this ocean world.

As described by Stern (2017): Any subsurface ocean biosphere is protected from lethal space environmental effects like vacuum, particle radiation, UV solar radiation, and meteor bombardment. However, the protective ice shell also masks any signs of life unless mechanisms bring them to the surface.

While there is an ongoing substantial investment in ocean world missions, there is not yet a parallel ocean worlds cross-integrated modeling effort in support of such missions.

As an example, plume modelers might submit a proposal in response to a R\&A Solar System Workings (SSW) solicitation, and the SSW would correctly categorize this proposal as an exosphere effort and thus place the proposal in an exosphere/atmosphere panel. A separate proposal on tidal heating, thermal flow, and surface temperatures on icy moons might find its way to a SSW geophysics/interior panel. A separate proposal examining the habitability of waterrock interfaces is relevant instead to the Habitable Worlds solicitation. A proposal on ocean fluid flow might be submitted to a solicitation outside of Planetary Science, e.g. within Earth Science. These proposals would be reviewed separately and no one would see the obvious, big-picture connection of one to the other - yet we know in OW studies that all of these topics are inseparably linked.

White Paper Objective: To emphasize the need for large scale 'floor-to-plume' integrated modeling efforts to improve our understanding of ocean worlds as complete systems. 
Linking In the Context of Astrobiology Research Coordinated Networks (RCN). Leaders of the Astrobiology program at $\mathrm{HQ}$ have recognized the need to form these linkages and have now included as a formal Research Coordinated Network the Network of Ocean Worlds (NOW). NOW integrates the leaders of Astrobiology consortia proposals with the PIs of topical R\&A proposals so as to catalyze new ideas and new exploration.

However, even within the RCNs, there is still not a formal attempt to update and merge models from the interior, to the ocean, to the plumes, and the eventual plume loss to the space environment. We take a parallel view as the RCNs but a more definitive view on the integration. Rather than connecting investigators, we recommend that Decadal Survey panels consider avenues for fostering the generation of integrated modeling systems that connect existing codes limited by their dominant focus. In doing so, we ensure that key modules are developed as part of a global model. This integrated modelling effort would have the added benefit of highlighting interface questions that are key to ocean worlds as a whole, but that would otherwise go unnoticed by studies limited to a particular component.

Examples of Analogous Integrated Modeling Efforts. There have been other large-scale analogous efforts in other fields to merge codes into a large integrated system. These include community-managed integrated ocean-atmosphere circulation models to study climate on Earth - like the Goddard Earth Observing System Version 5 (https://gmao.gsfc.nasa.gov/GEOS/). This Earth climate system can be configured for atmospheric circulation, ocean circulation, and chemistry transport and can also assimilate data inputs. While not as rigorously integrated, in heliophysics, NASA funds the Community Coordinated Modeling Center (CCMC) (https://ccmc.gsfc.nasa.gov/index.php) that houses various magnetoplasma codes of the solar wind and terrestrial magnetosphere. Team members are combining elements of these codes to create integrated products that can be used to predict the evolution of solar energetic particles created during coronal mass ejections from a flare source to the Earth-Moon system - which has predictive capability that is applicable to Human Exploration. The exoplant community has created a virtual planet simulator (VPLanet) that consists of codes that model planetary evolution (https://arxiv.org/abs/1905.06367). In a more tactical application, in studying the complex issue of spacecraft charging, NASA and USAF funded the development of the NASA Charging Analyzer Program (NASCAP) (https://software.nasa.gov/software/MFS-32056-1) that incorporates modules that simulate the space plasma environment and associated spacecraft surface charging - and modules to ingest the detailed digital spacecraft models.

In all of these cases, the sponsoring institution earmarked specific funds to integrate existing codes into a larger collection. The development of the integrated system has considerable expense due to transforming the codes into a common language, to be complied on a common platform, with module interfacing performed in a way that is seamless to the community user.

No analogous integrated product for modeling planetary oceans currently exists. Such a code would have to include modules like those shown in Figure 2 (described in more detail below in Section 2). Clearly, an effort for code integration goes well beyond an R\&A proposal, and possibly even beyond the funding in an RCN. It would have to be a devoted effort integrating modules from community-recognized authors. Since the effort would focus less on science and more on existing coding integration and platform compatibility, the RCN may not be the right venue for funding - but require a new, separate Ocean Worlds program element out of PSD. 


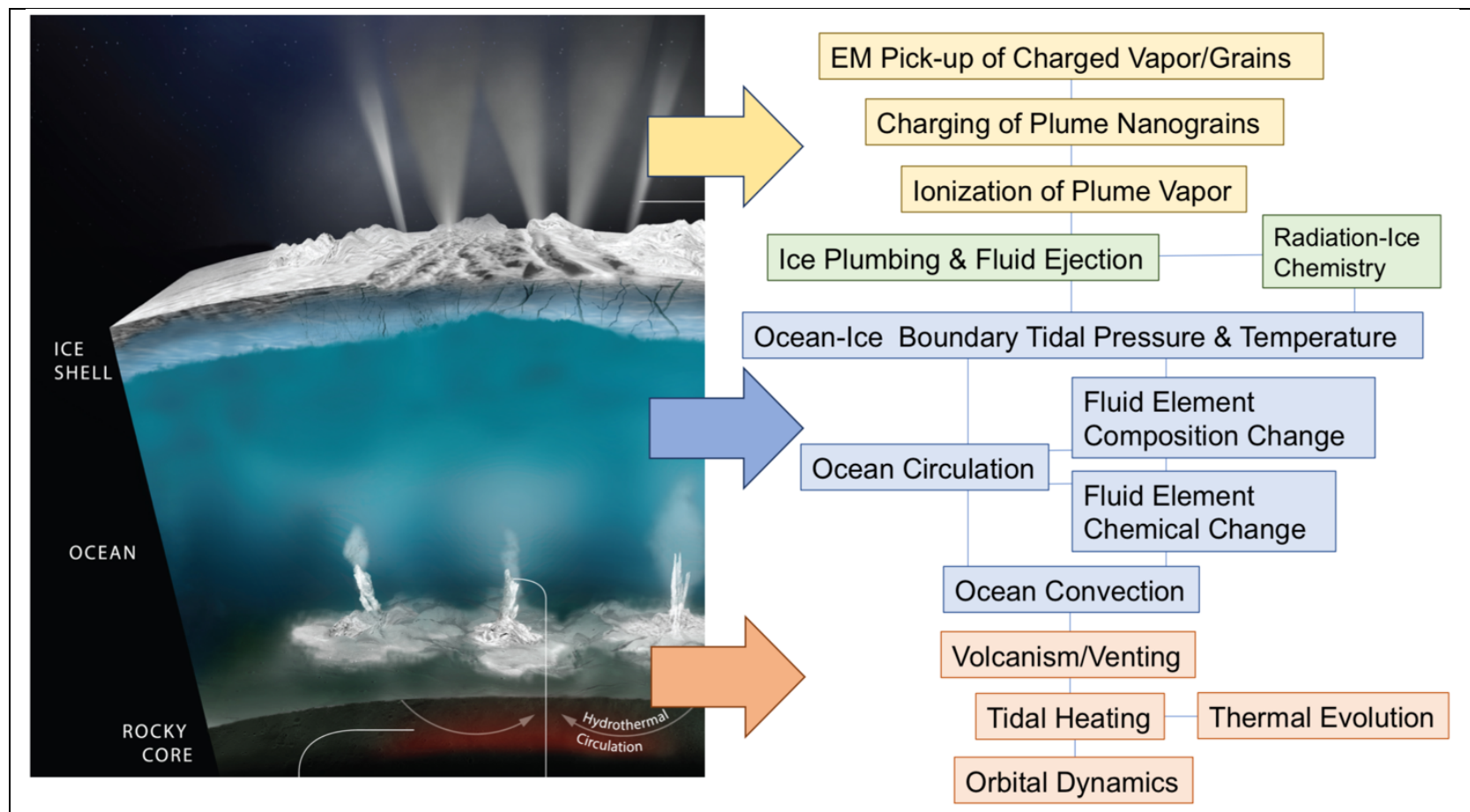

Figure 2- An overview of an integrated modeling approach combining modules of the core, ocean, and plume into one larger code.

\section{Merging Model Elements into an Integrated System}

As indicated in Figure 2, an integrated model would have a number of key modules that are normally developed in different areas of our community. Module complexity, including dimensionality (box, 1D, 2D, 3D) and underlying physics (e.g. ice fractures, hydrothermal vents), should match each other as well as match question scope. For instance, a 1D integrated model would be appropriate for the prediction of mean ocean composition, whereas a 2D (or 3D) model would be key for identifying a landing site based on the underlying ocean dynamics. Crucially, the community has not yet fully identified all key processes controlling the dynamics and habitability of ocean worlds and some processes will be difficult to incorporate in a global planetary model (as evidenced from e.g. the 30+-year struggle of parametrizing ocean processes in Earth System Models). The development of an integrated model, through iterations and increasing complexity, would help identify key fundamental science questions and limitations of numerical predictions, which would enable reliable guidance for NASA ocean worlds' missions. We now discuss several of the known outstanding science questions, which have implications for model development.

Core Energy Conversion. On the geology side, the thermal history/evolution and tidal forces in the deep interior would be calculated - these acting as the energy drivers for the entire process (Figure 3). Inputs may include orbital location, gravitational forces, and thermal gradients from any known thermal evolutional history. Given the change in gravitational forces, core deformation can be derived. Energy conversion from deformation processes into core surface thermal gradients will be necessary to calculate. We note that while there will be a regional determination on where thermal gradients are intense, the actual location of a vent likely will only be determined in a statistical way - similar to predicting a tornado location from a supercell. 
The conditions may be correct for venting, but the actual vent location requires other unique information like local geological stratigraphy, fault information, etc. The output would be a statistically-derived expectation of where a thermal vent might be located on the core-ocean interface, and thus sources of ocean convection.

Approaches to the Ocean Physics. Until more is known about extraterrestrial oceans, it may be of benefit to apply terrestrial ocean models for preliminary investigations of ocean worlds. This requires identifying and relaxing Earth-centric assumptions hard-wired into these models (as has been done in applying Earth climate models to exoplanets; see https://data.giss.nasa.gov/rocke3d/maps). For example, (1) ocean tides on ocean worlds are different from tides on Earth due to the overlying ice shell, and (2) the composition of ocean worlds can vary significantly from that of Earth oceans, which means that their respective dynamics may be qualitatively different. Ocean worlds may be depleted in salts compared to Earth oceans such that thermal convection, driven by e.g. the geothermal flux, may exist throughout the full water column and control exchanges with the solid core and the ice crust. Model developments and studies of convection in planetary liquid cores could prove helpful (more so than Earth ocean models in some cases) in designing the ocean module and coupling with the solid core (Nataf

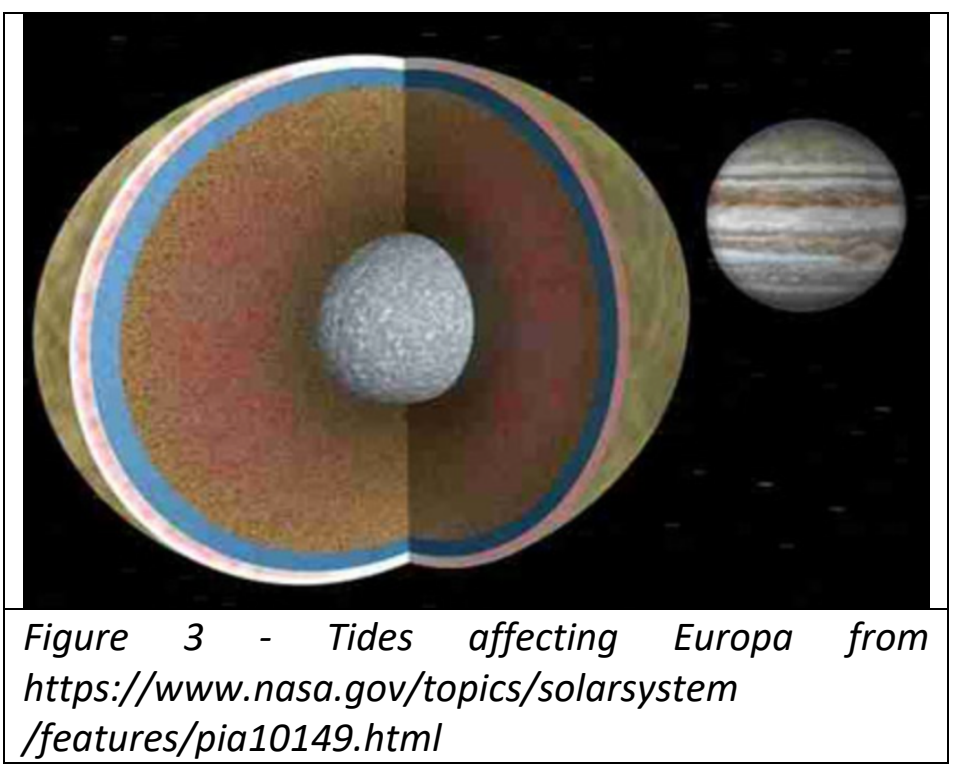
and Schaeffer, 2015).

The coupled evolution of an ice layer with an ocean, through melting/freezing of the iceocean interface, is an important process for Earth's climate (through evolution of ice shelf cavities and sea-level rise) and is likely similarly important (if not more) to ocean worlds. Incorporating ice-ocean interactions in Earth System Models is in its infancy but is also a top priority (IPCC 2019). Thus, there is an opportunity to work in collaboration with terrestrial scientists on the state-of-the-art coupling of ocean modules with ice modules.

More generally, in simulating ocean-solid interfaces, it may be valuable to use two complementary approaches: one that parameterizes a number of unknown but first-order properties (e.g. compositional and viscosity contrast, topography) so as to be able to rapidly explore a vast parameter space (millions of potentially meaningful cases, e.g. Tyler 2019), and one in which these properties are spatially resolved as a helpful benchmark in select cases (e.g. Chen et al. 2007).

Ocean Composition and Chemical Fluid Alteration. In a detailed ocean model, tracing compositional changes in fluid elements as they move from an internal ocean, to the surface, to a plume are needed to garner compositional and geological context of the reservoir. Assuming some initial salinity, temperature, and pressure, changes in temperature and pressure, and 
transport speed of the fluids, as functions of time, could be used to constrain their compositional changes en route to the surface.

The significance of exchange processes on the ocean worlds in our solar system is predicated on the possibility that fluids that are ejected onto the surface of these bodies are directly sourced from their internal oceans. For example, the geyser-like plumes that have been observed on Europa (Roth et al., 2014; Sparks et al., 2016; 2017) and Enceladus (Porco et al., 2006; Spencer et al., 2009) are believed to issue directly from their subsurface oceans.

However, theoretical modeling has shown that fluids erupted onto the surfaces of larger ocean worlds like Europa are more likely to originate in discrete crustal reservoirs that may or may not have a direct connection to the underlying ocean (Manga and Wang, 2007; Fagents et al., 2000; Fagents, 2003). Indeed, previous workers have shown that small reservoirs of liquid water likely exist perched within Europa's ice shell (Fagents, 2003; Johnston and Montési, 2014,

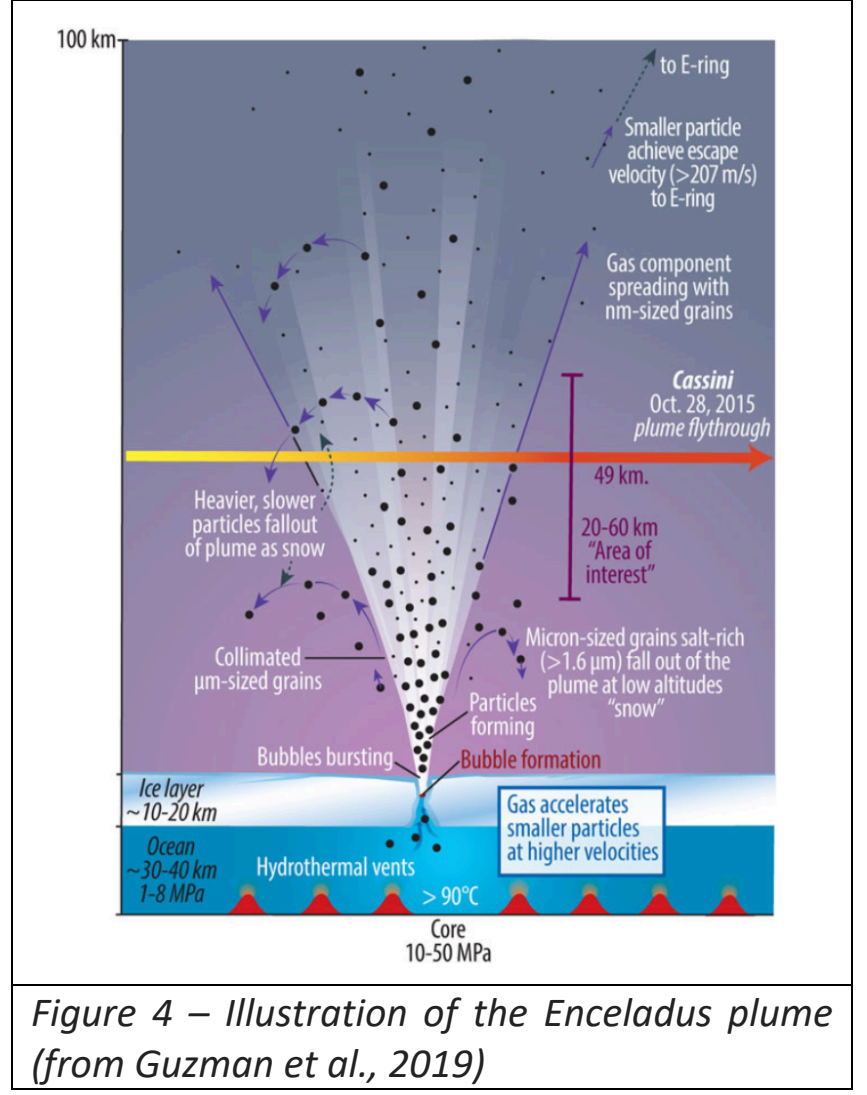
Craft et al., 2016; Culha and Manga, 2016; Manga and Michaut, 2017). Akin to magma plumbing systems on Earth, these reservoirs may be parts of complicated subsurface plumbing systems. The paths of fluids that are transported from these reservoirs to the surfaces of larger moons may therefore involve multiple episodes of diking and diapiric ascent (Quick and Marsh, 2016; Lesage et al., 2020).

Since fluids sourced from the ocean would travel farther (maximum distance of $30 \mathrm{~km}$ in the case of Europa) to arrive at the surfaces of large moons, they would undergo more cooling, which would lead to more precipitation and possibly a higher salt content than fluids that originate in fluid reservoirs that may reside at shallow levels in the crust. The composition (i.e., salt to water ratio) of fluids erupted onto Europa's surface could therefore be used as a means to determine where they

originated.

Thus, any model of fluid transport has to have a module to address these capillary-like effects in the fluid element's chemical and physical potency. Again, this might have to take on a statistical approach.

Melosh et al (2004) discussed the thermochemical properties of $\mathrm{NaCl}, \mathrm{MgCl}_{2}, \mathrm{Na}_{2} \mathrm{SO}_{4}$, and $\mathrm{MgSO}_{4}$ salt solutions of Europa's water ocean noting the importance of the effects of pressure on such systems. Manga and Wang (2007) modelled the eruption dynamics of liquid water based on subsurface ocean pressure on Europa and Enceladus, but they did not explore thermochemical changes in the water as it is erupted at the surface. Quick and Marsh (2016) investigated heat transfer in water as it moved through Europa's stagnant lid, while Lesage et al. 
(2020) investigated the dynamics associated with this transport. However, the dynamics and associated heat transfer of salt-rich fluids, and the change in their composition as a result of their movement through an ice shell has yet to be investigated.

Subsurface to Plume Connection. Theoretical modeling of planetary plumes (Figure 4) requires a systems-integrated approach because of the broad range of physical processes (chemical, dynamical, electromagnetic), phases (solid, fluid, gaseous) and flow regimes (fluid, rarefied, free molecular flow) encountered from the subsurface source out into the extended cloud. A plethora of analyses on Cassini data of the Enceladus plume are ideal examples of how in-situ and remote observations (Porco et al., 2006; Waite et al., 2006; Spitale and Porco, 2007; Spencer et al., 2006; Porco et al., 2014) of plume material can be used in models to infer subsurface properties (Tian et al., 2007; Smith et al., 2010, Tenishev et al., 2010; Dong et al., 2011; Hurley et al., 2015; Tucker et al., 2015; Yeoh et al., 2017). However, most of the analyses focus on particular regions and dynamics of the plume.

Currently our understanding of how the dynamics of the entire system are interrelated is relatively obscure. For example, there is a factor of 2 uncertainty of the Enceladus plume source rate derived between several of the different models referenced above (Goldstein et al., 2018). It is not clear whether this difference can be attributed to plume variability or if its related to modeling assumptions of the gas properties at the surface. It is well known that the source rate depends the subsurface properties which in turn govern the nucleation and acceleration of grains (Schmidt et al., 2008; Tucker et al., 2015), that are subsequently linked to the stratification in grain sizes of the plume (Hedman et al., 2009; Postberg et al., 2011) and ultimately controls the source of grains to the E-ring (Postberg et al., 2009) and source of charged nanograins to the magnetosphere (Smith et al., 2010; Hill et al., 2012; Mitchell et al. 2015).

The plume losses take the form primarily of electromagnetic pick-up of the charged species in the plume - like charged nanograins, plume ions, and electrons. Regarding the volatile flow field, the Knudsen number ( $K n=$ ratio of the mean free path-to-density gradient) which characterizes different flow regimes could be used to connect boundary conditions between relevant models. Specifically, the plumes may be collisional near the source, which would then increase the likelihood of species loss via plasma chemistry. For example, methane dissociates upon interaction with electrons between 10-100 eV, and these chemical alterations should be included in future codes - even if incorporated in a very simplistic fashion.

Radiolysis. High energy charged particles incident on the surface ice can alter the chemical state of the surface molecules - and this can especially affect the lifetime of any organic material that is emitted by the plume and falls back to the body. This radiolysis is especially important to consider for Europa that is immersed in the intense Jovian radiation belts. The effect is less of an issue at Enceladus since the intensity of high energy particles is substantially less. In any integrated code, modules that estimate species surface lifetime in a radiation environment should be included, since this represents a loss process for surface-residing organic material.

\section{Recommendations}

With a systems-integrated modeling approach, we integrate existing modeling pieces into one system to generate a larger model that garners an understanding of how the core and ocean variables affect the fluid physical and chemical end state. Obtaining a consistent set of boundary 
conditions between a system of integrated models would allow for more detailed analyses of processes such as fluid compositional changes with flow, jet intensity with orbital phase, stratification in grain sizes within plumes, ionization rates of the plume vapor and grains due to the local electromagnetic environment, conditions leading to formation of planetary coronae which are sources of material to the planetary system and other chemical and dynamical processes. Connecting the core-to-ocean-to-plume-to-ring in an integrated approach is the next logical step.

Funding: We propose that PSD set aside a set of funds to develop this integrated code for use by the entire community. As described above, we recognize that this is not a science endeavor per se, so funding via the astrobiology RCN is likely not appropriate - a separate funding line should be developed.

There are a set of examples in other fields in the development of integrated system models that have led to new successful findings, and adaptable for advancing the understanding when new information is found. A parallel concept is proposed herein.

First Steps: We also suggest forming Ocean Modeling Definition Team to identify the existing key pieces of an integrated system, missing pieces, and curators/developers of codes required to assemble that system. We then suggest the developers work with the OMDT to integrate codes, create common interfaces, and place the codes onto a common platform. The OMDT would identify where the platform would reside, who should create it, and recommend the repository operating system, etc. Like other NASA-developed large-scale models, the community would have access to run the codes to suit their particular application.

References: -Chen, C. (2007), J. Geophys. Res.,112, C03018. -Craft, K., et al. (2016), Icarus 274, 297-313.; -Culha, C., Manga, M. (2016), Icarus 271, 49-56.; -Dong, Y., et al. (2011), J. Geophys. Res., 116, A10204 Fagents, S.A., et al. (2000) Icarus 144, 54-88.-Fagents, S.A. (2003), J. Geophys. Res., 108, 5139.-Goldstein D. B. et al. (2018), In 'Enceladus and the Icy Moons of Saturn' p.175-194. -Guzman, M., et al. (2019), International J. of Astrobiology, 18, 47-51. -Hedman, M. M., et al. (2009), Astrophys. J. 693, 1749-1762.= Hill, T. W., et al. (2012), J. Geophys. Res. 117, A05209 -Hurley, D. M, et al. (2015), J. Geophys. Res., 120, 1763-1773. -IPCC (2019), IPCC Special Report on the Ocean and Cryosphere in a Changing Climate. Johnston, S. A., and Montési, L. G. J. (2014), Icarus 237, 190-201. -Lesage, E. et al. (2020) Icarus, In Press. -Nafat, H. C, and N. Schaeffer (2015), in Treatise in Geophysics, 8, 161-181. -Manga, M., Wang, C.-Y. (2007), Geophys. Res. Lett, 34, L07202. -Manga, M., Michaut, C. (2017), Icarus 286, 261-269. - Melosh, H.J., et al. (2004), Icarus 168, 498-502.- Mitchell, C. J., et al. (2015) Astronom. J., 149: 156. -Porco, C. C., et al. (2006), Science 311, 1393-1401.-Porco, C.C. et al. (2014), Astronom. J., 148:45. - Postberg, F., et al. (2009), Nature, 459 (7250): 1098-1101.-Postberg, F., et al. (2011), Nature 474 (7353): 620-622.-Quick, L.C., Marsh, B. D. (2016), J. of Volcanology and Geothermal Res., 319, 66-77.-Roth, L., et al. (2014), Proceedings of the National Academy of Sciences , 111,E5123-E5132 -Schmidt, J. N., et al. (2008), Nature 451 (7179): 685-88. -Smith, H. T., et al. (2010), J. Geophys. Res, 115, A10252. -Sparks, W. B., et al. (2016), Astrophys. J. 829, 121. -Sparks, W. B., et al. (2017), Astrophys. J. Lett., 839, L18. -Spencer, J. R., et al. (2009) In 'Saturn from Cassini-Huygens', Springer, New York, NY, pp. 683-724.-Spencer, J. R., et al. (2006), Science 311 (5766): 1401-5. -Spitale, J. N, and C. C. Porco (2007), Nature, 449 (7163): 695-697. -Stern, A., (2017), 49 ${ }^{\text {th }}$ AAS/DPS meeting (see also https://www.space.com/38577-fermi-paradox-alien-life-buried-oceans.html) -Tenishev, V., et al. (2010), J. Geophys, Res, 115, A09302 -Tian, F. A., et al. (2007), Icarus 188 (1): 154161. - -Tucker, O.J., et al (2015), Icarus, 257, 362-376. - Tyler R. (2019) 50 ${ }^{\text {th }}$ LPSC, abstract 2883. -Waite, J. H., et al., (2006) Science 311, 1419-22. -Yeoh, S. K. et al. (2017), Icarus, 281, 357-378. 